\title{
A Probe into Application Value of Ultrasonography in Clinical Diagnosis of Gynecologic Acute Abdomen
}

\author{
CUICUI LIU* \\ Department of Ultrasonography, Beijing Fengtai Hospital of Integrated Traditional Chinese and Western Medicine, Beijing \\ 100071, China
}

Liu et al.: Ultrasonography in Clinical Diagnosis of Gynecologic Acute Abdomen

\begin{abstract}
This paper aims to observe and analyze application value of ultrasonography in clinical diagnosis of gynecologic acute abdomen. The 180 cases of gynecologic acute abdomen were selected as the subjects. All the cases were examined by ultrasonography. The results of ultrasonography were compared with those of clinical diagnosis and then application effect and practical value of ultrasonography were analyzed. Among the 180 patients, ultrasonography revealed 80 cases, 6 cases, 4 cases, 10 cases, 12 cases, 40 cases, 24 cases of ectopic pregnancy, corpus luteum rupture, ovarian tumor torsion, acute uterine perforation, acute pelvic inflammatory disease, placenta previa and miscarriage, respectively. 176 cases were detected by ultrasonography, with coincidence rate of $97.78 \%$ compared to results of clinical examination. Ultrasonography is of great application value for gynecologic acute abdomen. During operation, ultrasonography is convenient and non-invasive, safe and reliable. It features high coincidence rate in examination, worthy of promotion in practice.
\end{abstract}

Key words: Gynecologic acute abdomen, diagnosis, ultrasonography, application value

At present, the continuous development of social economy leads to improvement of people's living standards, during which, people's living habits and diet are subject to changes, where health slowly affected. In obstetrics and gynecology, acute abdomen is a common disease. After developing the disease, patient's condition is complex and has rapid progress. A number of research data show that in recent years, number of China's cases of gynecologic acute abdomen has gradually increased, seriously plaguing the majority of women's physical and mental health ${ }^{[1]}$. Acute abdomen has multiple clinical manifestations, such as ectopic pregnancy (fig. 1), acute pelvic inflammatory disease and corpus luteum rupture (fig. 2), etc. Early diagnosis and identification of this disease as a basis to formulate scientific treatment program is essential. With the continuous development of medical technology, imaging examination technology has been continuously improved. Ultrasonography, with advantages of precision, rapidity and non-invasion, convenience, enjoys a wide range of applications in clinical gynecology and obstetrics and plays an important role $^{[2]}$. This study aims to observe and analyze application value of ultrasonography in clinical diagnosis of gynecologic acute abdomen. The 180 cases of gynecologic abdomen treated in our hospital from

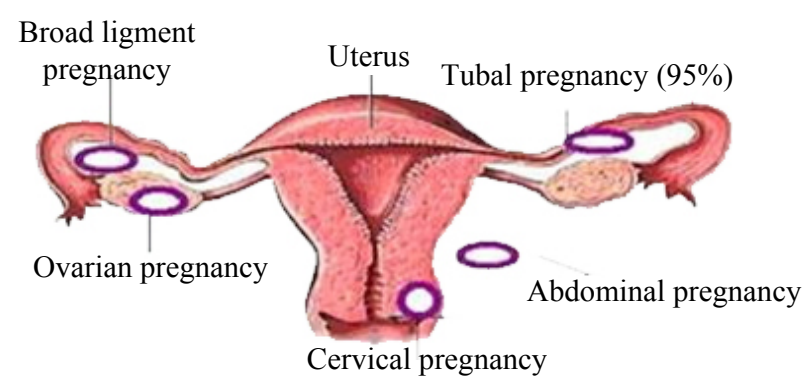

Fig. 1: Ectopic pregnancy

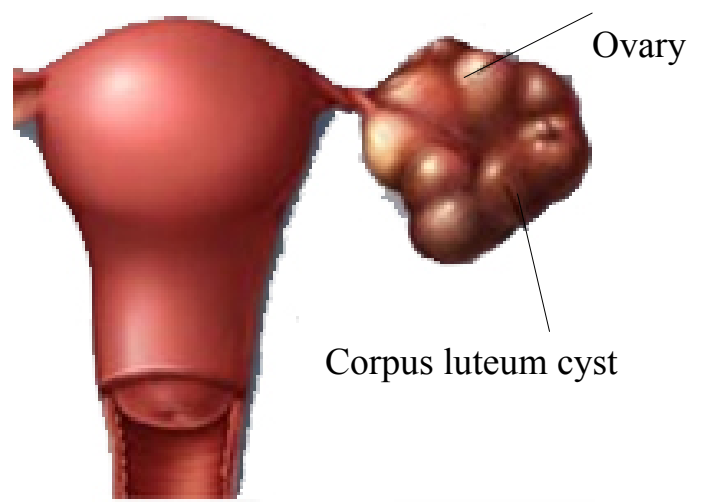

Fig. 2: Corpus luteum 
February 2014 to February 2017 were enrolled in this study. The age of the patients was between 24 and $52 \mathrm{y}$, with average age of $(30.8 \pm 2.5)$ y. Before and during admission, patients showed varying degrees of abdominal pain, including suddenly increased acute abdominal pain and chronic abdominal pain plus vaginal bleeding or vomiting and so on. The patients had the right to know and signed the relevant informed consent. The equipment used in this study was Hitachi ultrasonic unit with frequency of abdominal probe set. Its frequency in this study was controlled in the range of 3.5 MHz-6.0 MHz and frequency of vaginal probe was maintained in the range of $6.0 \mathrm{MHz}-9.0 \mathrm{MHz}$. Before the patient's abdominal examination, the bladder was in filling state. If there is an emergency, inject $0.9 \%$ normal saline in the examinee's bladder through catheter ${ }^{[3]}$. Before transvaginal exploration, the bladder fluid should be exhausted. During examination, conduct strict multi section examination and carefully observe size of the uterus, endometrial thickness, etc ${ }^{[4,5]}$. At the same time, whether there is mass around the patient's uterine attachment should also be closely observed. Moreover, substantially examine pelvic internal environment, to observe whether there is fluid problem. The data were analyzed and processed by SPSS 21.0 statistical software. The count data were expressed in (n, \%) and tested by chi square. Only when $p<0.05$ is satisfied, the difference is considered statistically significant. In this study, clinical pathology diagnosis showed there were respectively 81 cases, 7 cases, 4 cases, 12 cases, 12 cases, 40 cases, 24 cases of ectopic pregnancy, corpus luteum rupture, ovarian tumor torsion, acute uterine perforation, acute pelvic inflammatory disease, placenta previa and miscarriage. Ultrasonography revealed 80 cases, 6 cases, 4 cases, 10 cases, 12 cases, 40 cases and 24 cases of ectopic pregnancy, corpus luteum rupture (with ultrasound image shown in fig. 3), ovarian tumor torsion, acute uterine perforation, acute pelvic inflammatory disease (with ultrasound image shown in fig. 4), placenta previa and miscarriage, respectively. 176 cases were detected by ultrasonography. There was 1 case of corpus luteum rupture and 1 case of acute uterine perforation misdiagnosed plus 1 case of ectopic pregnancy and 1 case of acute uterine perforation missed in diagnosis. Compared with the results of clinical examination, coincidence rate of ultrasonography was $97.78 \%$ (Table 1). Gynecological acute abdomen has complex onset factors and rapid progress. The main clinical manifestation of the patient is abdominal pain. If not timely diagnosed and treated with active and effective

measures, it will increase probability of death ${ }^{[6]}$. Therefore, active application of effective ways to improve the diagnostic rate and implementation of effective treatment in the first time can significantly enhance success rate of treatment. According to the relevant data statistics, the most common onset factor of gynecological acute abdomen is ectopic pregnancy. In recent years, ectopic pregnancy has rising incidence, mostly tubal pregnancy (fig. 5) which accounts for about $95 \%\left[{ }^{[7]}\right.$. Ultrasonography shows full uterus, no intrauterine embryo or gestational sac. Also, endometrial decidual changes and abnormal uterine echo can be found. Exploration of pregnancy attachment area reveals mixed type of mass. Moreover, because of length of time, different occurrence site and different amount of bleeding, there are certain differences in ultrasonogram. In the early unruptured stage, complete fetal sac could be observed in the attachment area, plus fetal bud structure or fetal heartbeat. In this case, existence of ectopic pregnancy can be determined ${ }^{[8,9]}$. In early rupture, main clinical manifestation was mixed mass in complex structure. Color Doppler blood flow

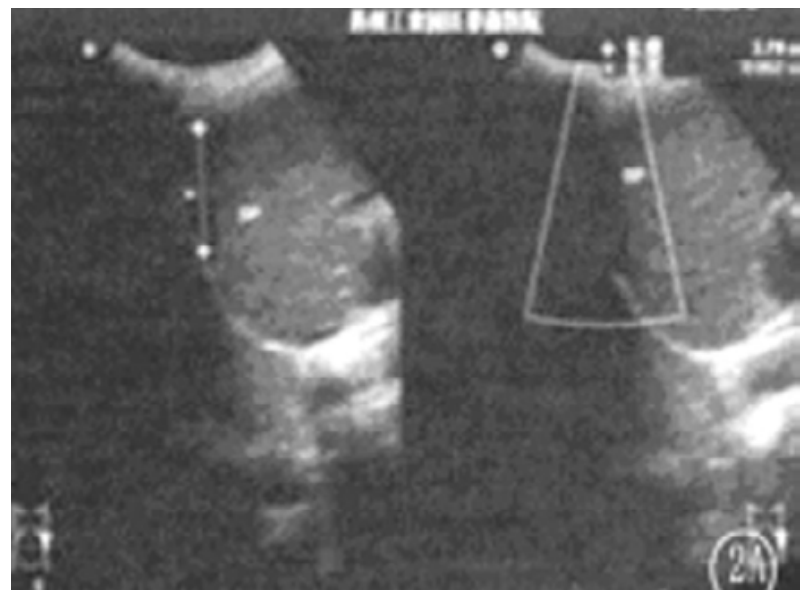

Fig. 3: Corpus luteum rupture

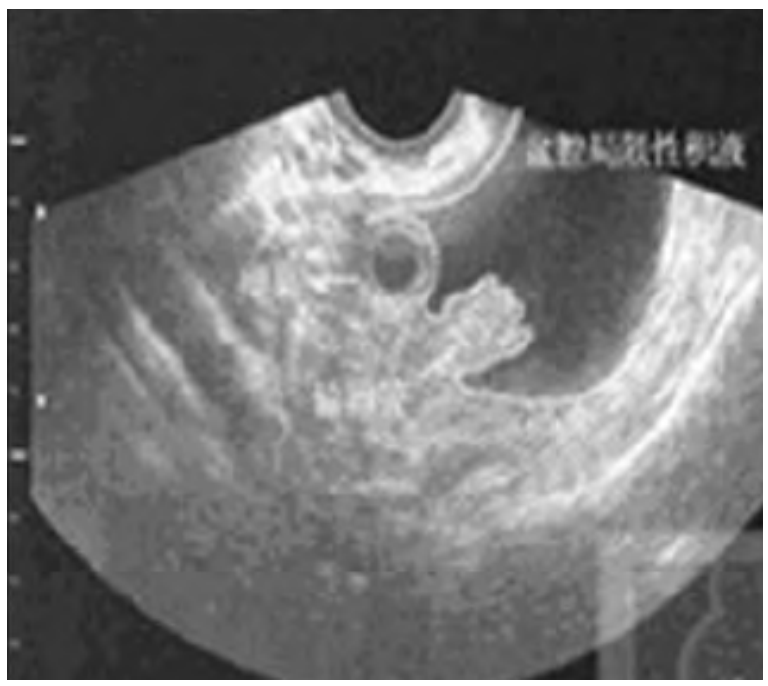

Fig. 4: Ultra sonogram of acute pelvic inflammatory disease
maceutical Sciences 
TABLE 1: DIAGNOSTIC COINCIDENCE RATE OF ULTRASONOGRAPHY [N (\%)]

\begin{tabular}{|c|c|c|c|c|c|}
\hline Disease type & $\begin{array}{c}\text { Clinical diagnosis } \\
\text { result }\end{array}$ & $\begin{array}{l}\text { Ultrasonography } \\
\text { result }\end{array}$ & Misdiagnosis & Missed diagnosis & $\begin{array}{c}\text { Diagnosis } \\
\text { coincidence rate }\end{array}$ \\
\hline Ectopic pregnancy & 81 & 80 & 0 & 1 & 98.77 \\
\hline Corpus luteum rupture & 7 & 6 & 1 & 0 & 85.71 \\
\hline Ovarian tumor torsion & 4 & 4 & 0 & 0 & 100.00 \\
\hline $\begin{array}{l}\text { Acute uterine } \\
\text { perforation }\end{array}$ & 12 & 10 & 1 & 1 & 83.33 \\
\hline $\begin{array}{l}\text { Acute pelvic } \\
\text { inflammatory disease }\end{array}$ & 12 & 12 & 0 & 0 & 100.00 \\
\hline Placenta previa & 40 & 40 & 0 & 0 & 100.00 \\
\hline Miscarriage & 24 & 24 & 0 & 0 & 100.00 \\
\hline Total & 180 & 176 & 2 & 2 & 97.78 \\
\hline
\end{tabular}

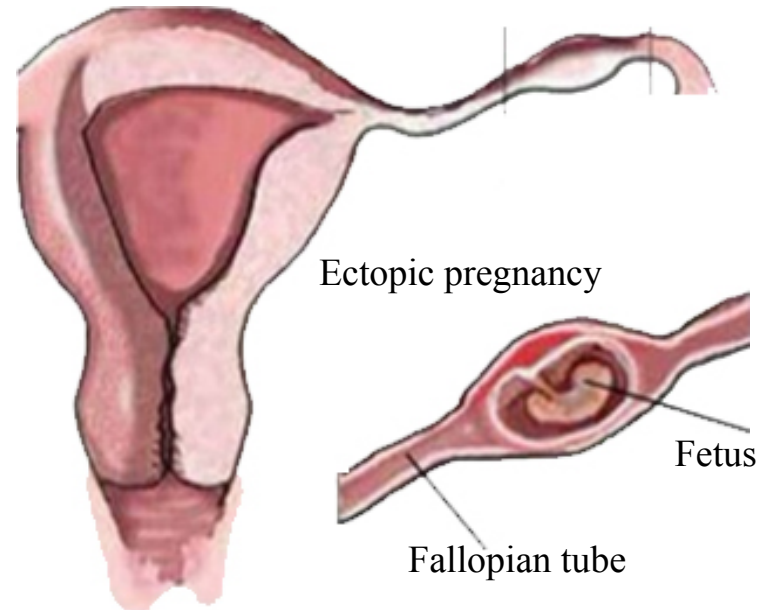

Fig. 5: Tubal pregnancy

display can clearly detect mass and peripheral blood flow signals. For patients with ovarian pregnancy, mass in embryo sac like structure and with abnormal ipsilateral ovarian morphology can be observed on the uterine side. In clinics, accurate judgment can be made based on irregular vaginal bleeding, acute abdominal pain symptoms and history of menelipsis ${ }^{[10]}$. In gynecological diseases, pelvic inflammatory disease and fallopian tube inflammation are common ones. In the early stage, patients have no typical clinical manifestations, but will show significant clinical symptoms and signs after further progress of the disease ${ }^{[11,12]}$. In ultrasonic examination, it can be observed that attachment area is accompanied by continuous tubular echo in different sizes, also there are vague, tortuous and thickened edges and oval shaped echo free zones can be found inside the pelvic cavity. Under normal circumstances, local mass can be observed for acute pelvic inflammatory disease cases and patients have significant pain, so clear clinical diagnosis can be made via ultrasound detection means based on patient's vital signs and disease history. For miscarriage cases, ultrasonoscopy can reveal full or increased uterus, collapsed gestational sac of irregular shape, but no fetal bud echo and fetal heartbeat. Uterine images of most miscarriage patients show irregular echogenic mass, which is due to partial decidual or placental retention under normal circumstances. Among female population during child bearing period, ovarian corpus luteum rupture has a high incidence, which also occurs in unmarried female. Its main clinical manifestations are nausea and vomiting, abdominal pain, etc. Also, relevant research data show that patients caused by corpus luteum rupture hemorrhage are owing to severe bleeding. Collapsed cyst with irregular profile can be observed through ultrasound image of corpus luteum rupture. Meanwhile, liquid dark area can be observed in sunken position of uterine rectum and bloody fluid can be extracted from vault after vagina puncture. In addition, cleardiagnosis viaultrasonography can be obtained for gynecologic acute abdomen such as miscarriage, acute pelvic inflammatory disease and placental abruption. At present, ultrasonography has been widely used in clinical examination. In operation, ultrasonography has good convenience, no trauma, higher security and reliability and can provide clearer images with higher lesion tissue resolution. At the same time, ultrasonography is affordable, so acceptable by the majority of patients. In this study, among 180 patients, ultrasonography revealed 80 cases, 6 cases, 4 cases, 10 cases, 12 cases, 40 cases, 24 cases of ectopic pregnancy, corpus luteum rupture, ovarian tumor torsion, acute uterine perforation, acute pelvic inflammatory disease, placenta previa and miscarriage, respectively. 176 cases were detected by ultrasonography, with coincidence rate of $97.78 \%$ compared to results of clinical examination and the imaging resolution was high. To sum up, for patients with gynecologic acute abdomen, implementation of ultrasonography can get higher diagnostic coincidence rate. Meanwhile, use of ultrasonography meets the current trend of development in medical technology, due to its convenient operation, non-invasion and low price. Hence, it can be widely 
applied in clinical diagnosis of gynecologic acute abdomen.

\section{Conflicts of Interest:}

The authors declared no conflict of interest.

\section{REFERENCES}

1. Sun Y, Zhang G. Diagnostic value of color doppler ultrasound in gynecological acute abdomen. Spec Health 2021;26:152

2. Cai ZY. Application value of color Doppler ultrasound in the diagnosis of gynecological acute abdomen. Contemp Med Forum 2021;19(1):121-2

3. Sun H. application of ultrasonography in clinical diagnosis of gynecologic acute abdomen. Contemp Med 2016;13:58.

4. Chen X. Application of ultrasonography in clinical diagnosis of gynecologic acute abdomen. For All Health 2016;22(7):197.

5. Miao B. Analysis of application of ultrasonography in clinical diagnosis of gynecologic acute abdomen. Med Forum 2016;22(5):646-7.

6. Zhao JP. Application analysis of ultrasound imaging technology in clinical diagnosis of acute abdomen in obstetrics and gynecology. Inner Mongol J Traditi Chin Med 2014;10:889.

7. Wang X, Ku M, De W, Qin L, Wa TB, LiP.Analysis of application of ultrasonography in clinical diagnosis of gynecologic acute abdomen. Chin J Mod Drug Appl 2013;11(15):51-2.
8. Zhao LM, Zhang GF. Application of three dimensional ultrasound in gynecological acute abdomen. Gen J Stomatol 2020;7(2):133-5.

9. Shang RM, Kong MG, Xu XW. Application value of transabdominal ultrasound combined with transvaginal ultrasound in the diagnosis of gynecological acute abdomen. Chin J Clin Ration Drug Use 2020;13(28):167-8

10. Wang XF. Study the clinical application value of abdominal ultrasound combined with vaginal ultrasound in the diagnosis of gynecologic acute abdomen. Women's Health Res 2020;(17):192-3

11. Hou CX. Study on the value of transvaginal ultrasound combined with abdominal ultrasound in the diagnosis of gynecological acute abdomen. Mod Med Imagelogy 2019;28(1):149-50.

12. Yan LM. Clinical application of ultrasonography in diagnosis and differential diagnosis of gynecological acute abdomen. J Imaging Res Med Appl 2018;2(23):143-4.

This is an open access article distributed under the terms of the Creative Commons Attribution-NonCommercial-ShareAlike 3.0 License, which allows others to remix, tweak, and build upon the work non-commercially, as long as the author is credited and the new creations are licensed under the identical terms

This article was originally published in a special issue, "Evolutionary Strategies in Biomedical Research and Pharmaceutical Sciences" Indian J Pharm Sci 2021:83(3) Spl issue;198-201 